\title{
Acute diabetic neuropathy following improved glycaemic control: a case series and review
}

\author{
N Siddique1, R Durcan1', S Smyth², T Kyaw Tun1, S Sreenan'1 and J H McDermott1 \\ 1Departments of Diabetes and Endocrinology, Connolly Hospital Blanchardstown, Royal College of Surgeons in \\ Ireland, Dublin, Ireland and 2Department of Neurology, Mater Misericordiae University Hospital, Dublin, Ireland
}

Correspondence should be addressed to $\mathrm{N}$ Siddique

Email

najiach@yahoo.com

\section{Summary}

We present three cases of acute diabetic neuropathy and highlight a potentially underappreciated link between tightening of glycaemic control and acute neuropathies in patients with diabetes. Case 1: A 56-year-old male with poorly controlled type 2 diabetes (T2DM) was commenced on basal-bolus insulin. He presented 6 weeks later with a diffuse painful sensory neuropathy and postural hypotension. He was diagnosed with treatment-induced neuropathy (TIN, insulin neuritis) and obtained symptomatic relief from pregabalin. Case 2: A 67-year-old male with T2DM and chronic hyperglycaemia presented with left lower limb pain, weakness and weight loss shortly after achieving target glycaemia with oral antihyperglycaemics. Neurological examination and neuro-electrophysiological studies suggested diabetic lumbosacral radiculo-plexus neuropathy (DLPRN, diabetic amyotrophy). Pain and weakness resolved over time. Case 3: A 58-year-old male was admitted with blurred vision diplopia and complete ptosis of the right eye, with intact pupillary reflexes, shortly after intensification of glucose-lowering treatment with an SGLT2 inhibitor as adjunct to metformin. He was diagnosed with a pupil-sparing third nerve palsy secondary to diabetic mononeuritis which improved over time. While all three acute neuropathies have been previously well described, all are rare and require a high index of clinical suspicion as they are essentially a diagnosis of exclusion. Interestingly, all three of our cases are linked by the development of acute neuropathy following a significant improvement in glycaemic control. This phenomenon is well described in TIN, but not previously highlighted in other acute neuropathies.

\section{Learning points:}

- A link between acute tightening of glycaemic control and acute neuropathies has not been well described in literature.

- Clinicians caring for patients with diabetes who develop otherwise unexplained neurologic symptoms following a tightening of glycaemic control should consider the possibility of an acute diabetic neuropathy.

- Early recognition of these neuropathies can obviate the need for detailed and expensive investigations and allow for early institution of appropriate pain-relieving medications.

\section{Background}

Diabetic neuropathy is the most prevalent chronic complication of diabetes mellitus (DM). Diabetic neuropathy most commonly presents as a distal sensorimotor neuropathy, where reduced or absent protective sensation in the lower limbs leads to an increased risk of foot ulcers and peripheral neuropathic pain is common. Diabetic neuropathy can also present as acute neuropathies, however, in this article we present 
three of the less common acute presentations of diabetic neuropathy and discuss appropriate investigations, treatment and prognosis. We also highlight an important and potentially underappreciated link between tightening of glycaemic control and acute diabetic neuropathies.

\section{Case presentation}

\section{Case 1}

A 56-year-old man presented to the Emergency Department complaining of an 8-month history of fatigue, polyuria, polydipsia, blurred vision and weight loss. Physical examination was normal, with a BMI of 21 $\mathrm{kg} / \mathrm{m}^{2}$. Laboratory testing revealed a HbA1c of $145 \mathrm{mmol} /$ mol (15.4\%), confirming a diagnosis of DM. Basal-bolus insulin therapy was commenced. Six weeks later the patient returned to the hospital complaining of chest, shoulder, arm and leg pain with associated paraesthesia. The pain was described as severe, and he reported that light touching of his skin was extremely painful. He described further weight loss of approximately $10 \mathrm{~kg}$ since the first visit. Glucose readings had improved on insulin, with readings between 5 and $7 \mathrm{mmol} / \mathrm{L}$. HbA1c had fallen to $55 \mathrm{mmol} / \mathrm{mol}$.

\section{Investigation case 1}

Endoscopic examination of the stomach and duodenum did not show significant pathology, and other routine investigations were unremarkable.

\section{Treatment case 1}

Treatment-induced neuropathy ('insulin neuritis') was suspected, and pregabalin was prescribed.

\section{Outcome and follow-up case 1}

Weight increased and symptoms resolved on follow-up.

\section{Case 2}

A 67-year-old man presented with a 1-year history of fatigue, polyuria, polydipsia, blurred vision and $7 \mathrm{~kg}$ weight loss. Physical examination revealed a BMI of $28 \mathrm{~kg} /$ $\mathrm{m}^{2}$ and reduced peripheral vibration and monofilament sensation on both feet. Random blood glucose was elevated at $34 \mathrm{mmol} / \mathrm{L}$ and HbA1c was $140 \mathrm{mmol} / \mathrm{mol}$ (15.0\%), confirming a diagnosis of DM. Basal-bolus insulin was instituted and resulted in resolution of hyperglycaemia and osmotic symptoms. Insulin was subsequently discontinued, and he was discharged on metformin $1000 \mathrm{mg}$ twice a day and gliclazide modified release of
$30 \mathrm{mg}$ a day. Three months later, glucose readings were consistently below $6 \mathrm{mmol} / \mathrm{L}$ and without documented hypoglycaemia. The patient then complained of a new symptom of severe left-sided groin pain radiating down his left leg. There was associated left leg weakness, such that he walked with a stick. He had lost a further $3 \mathrm{~kg}$ in weight despite good glycaemic control. Examination revealed weakness of left hip flexion and left knee extension, an absent left patellar reflex and an atrophied left quadriceps. HbA1c checked 2 months later returned at $42 \mathrm{mmol} / \mathrm{mol}$, indicating excellent glycaemic control.

\section{Investigation case 2}

Nerve conduction studies and electromyography (EMG) were performed. Results were suggestive of a left-sided lumbosacral plexopathy most severely affecting the quadriceps muscles, supporting the clinical diagnosis of DLRPN (diabetic amyotrophy).

\section{Treatment case 2}

Pregabalin was instituted, and physiotherapy was advised.

\section{Outcome and follow-up case 2}

Over time his pain resolved and he discontinued the pregabalin. One year later he continued to walk with a stick due to ongoing leg weakness, but at 2 years following initial presentation, he could walk unaided. Weight had increased by $10 \mathrm{~kg}$ since the initial presentation with DLRPN.

\section{Case 3}

A 58-year-old man complained of double vision on a background of type 2 diabetes diagnosed 2 years prior to presentation. Three months prior to the current presentation, as a result of poorly controlled diabetes, his general practitioner had commenced dapagliflozin, and the patient had increased his exercise levels and adherence to dietary advice. Glucose readings in the weeks prior to his presentation had fallen into the normal range and HbA1c was $46 \mathrm{mmol} / \mathrm{mol}$, indicating excellent glycaemic control. On examination, there was an almost complete ptosis of the right eyelid. Pupillary reflexes were intact. The right eye was fixed in the 'down and out' position indicating an oculomotor nerve palsy with intact cranial nerves IV and VI.

\section{Investigation case 3}

As investigations to rule out alternate pathology including MRI brain, anti-muscarinic and anti-acetylcholine receptor antibodies, anti-nuclear factor, anti-neutrophil cytoplasmic antibodies and erythrocyte sedimentation 
rate were normal, a diagnosis of a pupil-sparing third nerve palsy secondary to diabetic mononeuritis was made.

\section{Treatment case 3}

He was continued on the aforementioned medications and advised for close monitoring of blood sugars.

\section{Outcome and follow-up case 3}

Three months later the patient's glycaemic control remained satisfactory, and his ophthalmoplegia had resolved.

\section{Discussion of 3 cases}

The most common presentation of diabetic neuropathy, DSN, is a diffuse and nerve-length-dependent neuropathy. The pathophysiology of DSN is multifactorial but reasonably well understood, and the link between poor glycaemic control and DSN is well established. Optimisation of glycaemic control significantly reduces the relative risk of DSN in T1DM (1) and in T2DM (2). Symptoms of DSN are typically chronic and irreversible, but are frequently mild to the extent that some patients may present without symptoms - that is, the neuropathy is discovered on clinical examination.

The acute neuropathies experienced by the three patients we describe are an altogether different proposition: rare, acute, severe but reversible, not occurring as a consequence of chronic hyperglycaemia and poorly understood.

In this paper we present three cases of acute diabetic neuropathy - treatment-induced neuropathy (insulin neuritis), diabetic lumbosacral radiculoplexus neuropathy (diabetic amyotrophy) and a pupil sparing oculomotor nerve palsy. While all three acute neuropathies have been well described in the past, all are rare and hence require a high index of clinical suspicion, as diagnosis rests largely on recognizing the symptoms and signs in the appropriate context and excluding alternative pathology.

All three of our cases are linked by the acute development of neuropathy in patients who had achieved a significant improvement in their glycaemic control prior to the development of the acute neuropathy. In the first case this improved control was achieved with insulin, in the second case with insulin followed by metformin and a sulphonylurea and in the third case with an SGLT-2 inhibitor and lifestyle changes added to metformin.

An association between improved glycaemic control and acute worsening of diabetic retinopathy is well described (1), but a corresponding link between tight glycaemic control and acute neuropathies less well so.
A link between acute lowering of blood glucose and acute neuropathies has been best described in cases of TIN and is reflected in the naming of the condition, but an association between tightening of glycaemic control and onset of DLPRN has not been previously highlighted. It is difficult to adequately scrutinize prior case series of DLRPN as the publications are typically from the era when assessment of glycaemic control rested on detection of glycosuria. When considering a potential link between acute lowering of blood glucose and DLRPN, it is interesting to review the three seminal cases described in detail by Casey et al. (3), however. In one patient the symptoms developed shortly after starting insulin treatment - a point not highlighted by the authors at the time as being relevant to the presentation. A second patient had insulintreated diabetes and developed symptoms shortly after an admission to hospital with chest pain - again, in view of our contention that an acute tightening of glycaemic control might trigger an onset of DLRPN, it is tempting to speculate that the hospital admission resulted in an acute improvement in glycaemic control in this patient.

Why might an acute lowering of blood glucose cause an acute neuropathy? A small number of research papers have attempted to answer this question, largely prompted by the association between acute neuropathy and cases of TIN. Ohshima et al., in a rat model, found reduced neural blood flow during $3 \mathrm{~h}$ of hypoglycaemia induced by the administration of insulin injections $(4,5)$. Repeated induction of hypoglycaemia resulted in endothelial swelling of endoneural microvessels on histological examination $24 \mathrm{~h}$ following the hypoglycaemic events. Other authors have suggested that apoptosis of axons can occur following sudden glucose deprivation: using an in vitro model of dissociated rat dorsal root ganglions (DRG), Honma et al. found that neurons maintained in hypoglycaemic medium were less able to withstand exposure to acute hypoxia than neurons maintained in hyperglycaemic medium (6). Hypoxic conditions resulted in apoptosis of DRG neurons when maintained in hypoglycaemic medium.

None of the three patients we describe had hypoglycaemia per se, nor was documented hypoglycaemia present in any of the six patients in the largest case series of TIN (7). It could be argued, however, in a patient with prolonged severe hyperglycaemia, that a rapid drop to normal glucose levels could result in a relative hypoglycaemia - with osmotic shifts causing the same damage to the endoneural- microvessels as those described in the experimental conditions described in animal models.

The findings in the research papers referenced previously, the clear clinical correlation between improved 
glycaemic control and TIN in case reports and the clinical course in the patients we describe all lead us to postulate a link between a relative hypoglycemia - caused by rapid reduction in glucose levels in patients with chronic hyperglycaemia - and the development of acute diabetic neuropathies. These neuropathies could be considered, therefore, to be iatrogenic, and their onset may prompt consideration of a period of 'permissive hyperglycaemia' to improve symptomatology. In the first case of TIN described by Caravati in 1933 (8), the patient's pain was refractory to analgesics and sedatives, but resolved within 3 days of stopping insulin - attempts to reintroduce insulin were met with similar levels of pain. Gibbons et al. propose limiting the fall in $\mathrm{HbA} 1 \mathrm{c}$ to $<2 \%$ over a 3-month period to prevent TIN (9), and we propose that this advice could apply equally to the other acute neuropathies we describe - but whether allowing permissive hyperglycaemia is the correct approach to adopt when patients develop symptoms remains a matter for debate.

Clinicians caring for patients with diabetes who develop otherwise unexplained neurologic symptoms following a tightening of glycaemic control should consider the possibility of an acute diabetic neuropathy. Early recognition of these neuropathies can obviate the need for detailed and expensive investigations, allow for early institution of appropriate pain relieving medications and could prompt consideration of a period of permissive hyperglycaemia.

\section{Declaration of interest}

The authors declare that there is no conflict of interest that could be perceived as prejudicing the impartiality of the research reported.

\section{Funding}

This research did not receive any specific grant from any funding agency in the public, commercial or not-for-profit sector.

\section{Patient consent}

Written informed consent has been obtained from the patients for publication of the submitted article.

\section{Author contribution statement}

N Siddique, R Durcan, S Smyth, T Kyaw Tun, S Sreenan and J H McDermott were involved in the diagnosis and management of all cases in this case series and they have contributed to writing and reviewing this manuscript.

\section{References}

1 Diabetes Control and Complications Trial Research Group, Nathan DM, Genuth S, Lachin J, Cleary P, Crofford O, Davis M, Rand L \& Siebert C. The effect of intensive treatment of diabetes on the development and progression of long-term complications in insulin-dependent diabetes mellitus. New England Journal of Medicine 1993329 977-986. (https://doi.org/10.1056/ NEJM199309303291401)

2 Ismail-Beigi F, Craven T, Banerji MA, Basile J, Calles J, Cohen RM, Cuddihy R, Cushman WC, Genuth S, Grimm RH, et al. Effect of intensive treatment of hyperglycaemia on microvascular outcomes in type 2 diabetes: an analysis of the accord randomised trial. Lancet 2010376 419-430. (https://doi.org/10.1016/S0140-6736(10)60576-4)

3 Casey EB \& Harrison MJ. Diabetic amyotrophy: a follow up study. BMJ 19721 656-659. (https://doi.org/10.1136/bmj.1.5801.656)

4 Ohshima J \& Nukada H. Hypoglycaemic neuropathy: microvascular changes due to recurrent hypoglycaemic episodes in rat sciatic nerve. Brain Research 2002947 84-89. (https://doi.org/10.1016/s00068993(02)02910-4)

5 Ohshima J \& Nukada H. Experimental hypoglycemic neuropathy in rat: nerve blood flow and nerve conduction study St. Marianna. Medical Journal 2006348591.

6 Honma H, Podratz JL \& Windebank AJ. Acute glucose deprivation leads to apoptosis in a cell model of acute diabetic neuropathy. Journal of the Peripheral Nervous System 20038 65-74. (https://doi. org/10.1046/j.1529-8027.2003.03009.x)

7 Dabby R, Sadeh M, Lampl Y, Gilad R \& Watemberg N. Acute painful neuropathy induced by rapid correction of serum glucose levels in diabetic patients. Biomedicine and Pharmacotherapy 200963 707-709. (https://doi.org/10.1016/j.biopha.2008.08.011)

8 Caravati C. Insulin neuritis: a case report. Virginia Medical Mon 1933 $59745-746$.

9 Gibbons CH \& Freeman R. Treatment-induced neuropathy of diabetes: an acute, iatrogenic complication of diabetes. Brain 2015 138 43-52. (https://doi.org/10.1093/brain/awu307)

Received in final form 19 December 2019 Accepted 14 January 2020 